\title{
Histomorphological and Immunohistochemical Study of Extra Nodal Lymphoma
}

\author{
B R Vani, Sandhyalakshmi BN, Netra M Sajjan*, Panduranga C, Deepak kumar B and Srinivasamurthy V \\ Department of Pathology, ESICMC \& PGIMSR, Bangalore
}

\begin{abstract}
Background: NHL are heterogeneous group of hematolymphoid malignancy occurring in nodal and extra nodal sites. Extra nodal lymphoma (ENL) is distinct from nodal lymphomas due to varied anatomical location, morphological diversity.

Methods: A retrospective observational study was conducted in the department of pathology from January 2011 till December 2019. Clinical details and other investigations were recorded. Gross morphological features were noted. Slides are reviewed microscopically and blocks were subjected to IHC for further typing cases were selected as per Dawson et al criteria.

Result: During study period, ENL constituted 25 cases (29.4\%) of the 85 cases of all NHL. Age range is 05 to 63 years with a mean age of 42.5 years. Male to female ratio is $2.2: 1$. Head and neck is the most common site of ENHL constituting 19 cases (76\%), next commonest location is in the GIT with 3 cases (12\%). We had one each case of ENHL in testis, breast and spine (4\% each). On IHC, $92 \%$ cases $(23$ cases) exhibited $\mathrm{B}$ cell phenotype and the remaining $08 \%$ (02 cases) were of $\mathrm{T}$ cell phenotype.

Conclusion: Lymphomas in the extra nodal sites are rare but has the propensity to occur in any anatomic site. Hence a differential of lymphoma to be considered in extra nodal site. Histology with IHC enables to differentiate from poorly differentiated carcinoma as the treatment modalities and prognosis varies.
\end{abstract}

Keywords: Extra Nodal Lymphoma, Non-Hodgkin's lymphoma, IHC, Histopathology.

\section{Introduction}

Non-Hodgkin's lymphoma (NHL) is heterogeneous group of hematolymphoid malignancy occurring in nodal and extra nodal sites. Extra nodal Non-Hodgkin's Lymphoma (ENHL) forms one fourth of all NHL, constituting 24$48 \%$. ENHL are distinct from nodal lymphomas due to varied anatomical location. They are widely distributed throughout the body tissues, arise in any anatomical site such as head and neck, skin, central nervous system (CNS), bone, thyroid, breast, orbit, testis, adrenals, pancreas, genitourinary tract and ovary with commonest being gastrointestinal tract (GIT) ${ }^{[1,2,3]}$. Krol et al liberally considered primary ENHL as all patients presenting with NHL apparently originated at extra nodal sites even in the presence of disseminated disease; provided ENHL site was clinically dominant ${ }^{[2]}$.

Etiology is multifactorial which includes viral and bacterial infections. Immunosuppressive therapy, exposure to pesticides and environmental agents are implicated in disease development in addition to local factors ${ }^{[2,3]}$. Also, extra nodal lymphoma (ENL) exhibits varied clinical presentation, morphological diversity and aggressive behavior. Being clonal neoplasms of Non-Hodgkin's; ENL has many immunohistological subtypes, prompting further characterization and diagnosis to promote targeted therapy since clinical outcomes vary. ${ }^{[4]}$ Increasing trend in incidence of ENL is observed in past two decades when compared to nodal lymphomas. ${ }^{[1,2,5]}$ In this background the present study was undertaken to evaluate the prevalence, clinical features, histomorphology and immunohistochemistry of extra nodal NHL and to understand tumor biology.

\section{Materials and Methods}

A retrospective observational study was conducted on all diagnosed cases of ENL in the dept of Pathology, ESIC Medical College \& PGIMSR, Rajajinagar. Duration of the study was nine years from January 2011 till December 2019. Clinical details were collected from department case files and from the medical records. Details of investigations done such as peripheral smear, bone marrow aspiration/ biopsy, chest X ray, CT scan, PET scan to look for lesions elsewhere, were collected. Gross morphological features of excised or biopsied specimens were noted. Histopathology slides, immunohistochemistry (IHC) slides were retrieved and reviewed microscopically. Immunohistochemistry was performed on formalin fixed paraffin blocks using peroxidase-antiperoxidase method with secondary antibodies from Biogenex manufacturer. Markers of CD45, CD20, CD 79a, BCl-2, BCL-6, CD3, CD5, CD 10 were used and evaluated. Based on differentials in HPE, additional IHC markers were incorporated for diagnosis, as 
per the case requirement such as CK, EMA, chromogranin etc. ENL cases were selected as per Dawson et al criteria characterized by absence of palpable superficial lymph node on first examination, absence of mediastinal lymphadenopathy on plain $\mathrm{x}$ ray chest, with dominant lesion at extra nodal site and involvement of lymph nodes at vicinity, while WBC within normal range ${ }^{[2]}$. Inclusion of tonsil of Waldeyer's ring as nodal or extra nodal NHL is debatable. In the present study tonsillar lesions were included similar to study done by Mishra $\mathrm{P}$ et al ${ }^{[2]}$. Primary nodal NHL, plasma cell neoplasms, leukemic cases were excluded from the study. ENL cases were classified as per WHO classification of tumors of hematopoietic and lymphoid tissues 2017 Revised edition classification, and clinical staging done according to Ann Arbor classification. Data was analyzed using descriptive statistics such as mean, median and range.

\section{Result}

During study period, out of 85 cases of all NHL, ENL constituted 25 cases (29.4\%). Age range was 05 to 63 yrs with a mean age of occurrence being 42.5 years. Majority are males (17 cases); with male to female ratio being 2.2:1. Grossly, the cut surface of the excised lesions is grey white and fleshy with rubbery consistency in few. Head and neck are the most common site of ENHL constituting 19 cases (76\%). Of the head and neck ENHL, tonsillar, orbital, oral, paranasal sinus lesions constituted 57.9\% (11 cases), 21\% (4 cases), 15.8\% (3 cases), and $5.3 \%$ (1 case) respectively. The next commonest location is in the GIT constituting 3 cases $(12 \%)$. We had 1 case of ENHL in testis, breast and spine (4\% each). On IHC, 92\% cases (23cases) exhibited $\mathrm{B}$ cell phenotype and the remaining $08 \%$ (02 cases) were of $\mathrm{T}$ cell phenotype. The most common histology among $\mathrm{B}$ cell lymphoma is diffuse large B cell lymphoma, not otherwise specified (DLBCL, NOS) accounting to 19 cases (82.6\%); others are follicular lymphoma (03 cases $13 \%)$ and small cell lymphoma(1 case). T cell lymphoma (02 cases) were confined to gingivobuccal sulcus of oral cavity, paranasal sinus and all exhibited histology of diffuse large cell lymphoma.

\section{Discussion}

ENHL is a heterogenous disorder with regard to geographical and ethnical diversities, hence frequency varies in different parts of world. ${ }^{[2]}$ Occurrence of ENHL in western countries is $24-48 \%$ of the NHL. Incidence of ENHL in Asian countries is $42 \%$ in Pakistan, $45 \%$ in Kuwait, $46.6 \%$ in, Japan, $55 \%$ in Korea, $58.5 \%$ in Thailand and $44.9-61.4 \%$ in China. ${ }^{2]}$ In the present study ENHL is $29.4 \%$ and slightly lower compared to other Asian countries.
In lymphomas, the genomically unstable germinal/post germinal center B cells undergo somatic hypermutation more frequently than $\mathrm{T}$ cells attributing to the increased prevalence of B cell neoplasms. Prevalence of B cell ENHL is $53-82 \%$. In our study B cell phenotype is $92 \%$ and DLBCL-NOS histology seen in $82.6 \%$ cases, similar to various studies reported in literature. ${ }^{[1,2,7,8]}$

Peak incidence of ENHL is in $4^{\text {th }}$ to $5^{\text {th }}$ decade with age range of 2-75 years and more in males. ${ }^{[2,9]}$ In the present study, age range is 05-63 years, and more cases were seen in the $5^{\text {th }}$ decade. Male preponderance with M:F ratio of 2.1:1 observed and is comparable to various studies reported in literature. ${ }^{[1,2,10]}$ Also all the 25 cases of ENHL were immunocompetent and had no B symptoms, but however presented with signs and symptoms pertaining to location.

Primary ENL are diverse group of disorders arising from various tissues other than lymph nodes. ${ }^{[1,10]}$ Currently term extra nodal lymphoma refers to lymphoma with no /only minimal lymph node involvement and with clinical dominant extra nodal component. ${ }^{[4]}$ ENL arise in almost any organ, and also in sites devoid of lymphocytes. ${ }^{[4]}$ However, term ENL is controversial/ambiguous with some studies histologically considering tonsil and Waldeyer's ring as extra nodal ${ }^{[2]}$ while others exclude it completely. ${ }^{[1,8]}$ GIT, skin, bone, breast are most common sites of ENL, and GIT lymphomas constitute $30-40 \%$ of ENL. ${ }^{[4]}$ If Waldeyer's ring lymphomas are included then head and neck is the $2^{\text {nd }}$ most frequent location. ${ }^{[1,4,11]}$ and of it tonsillar lesions are the commonest. ${ }^{[2,4]}$ Other sites affected in Waldeyer's ring are nasopharynx and the base of tongue. ${ }^{[4]}$

Literature search reveals varied occurrence of head and neck lymphoma ranging from $10 \%-20 \%$ and up to $70 \%$ occurs in the palatine tonsil. ${ }^{[2,7]}$ However, Mishra P et al in their study observed, head \& neck was the most common site of ENL constituting $36 \%$ of adult population. ${ }^{[2]}$ In the present study also head and neck is the most common site of ENL with tonsillar ENL, the commonest (57.9\%). Next common location is orbital ENL (21\%) and our observation is in concordance with Mishra P et al. ${ }^{[2]}$

Peak incidence in tonsils is seen in 6-7 decade with male preponderance. ${ }^{[7,12]}$ In our study, tonsillar ENL is more in $4^{\text {th }}$ and $5^{\text {th }}$ decade with male: female ratio being $1.8: 1$. High index of suspicion needed as tonsillar ENL presents as progressive sore throat, partially relieved on treatment. ${ }^{\text {[7] }}$ Clinically, B symptoms in tonsillar ENL often develop late in advanced disease, and lesions more than $7 \mathrm{~cm}$ exhibit poor outcome. Most of tonsillar lymphoma are B cell phenotype with 67-96 \% exhibiting DLBCL histology and DLBCL is often aggressive. ${ }^{[7,12]}$ High grade transformation of pre-existent low-grade mucosa associated lymphoid 


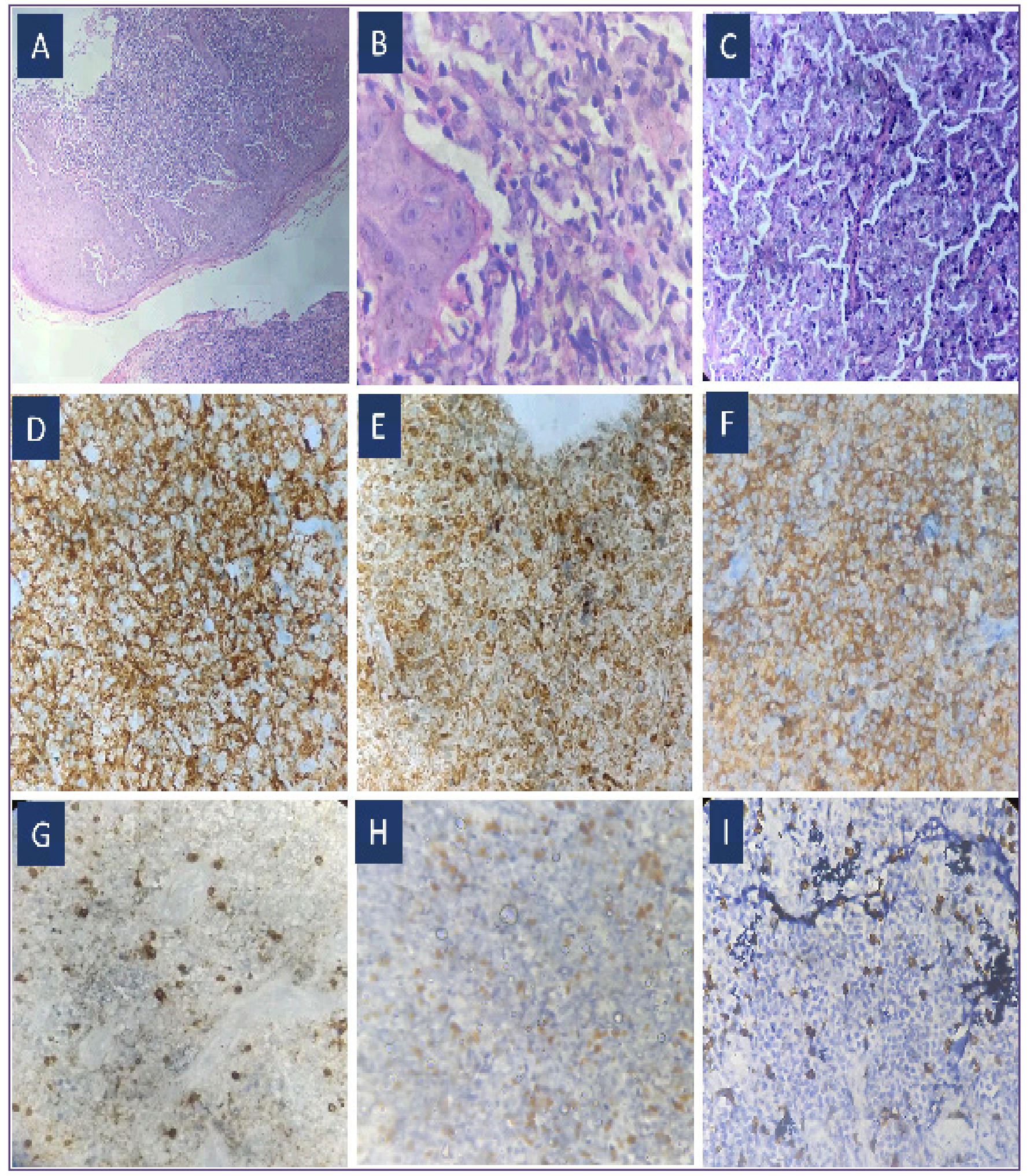

FIG. 1: (A, B \& C) Microphotograph of tonsil showing Diffuse large cell lymphoma (H\&E 10x,40x,100x). Fig D showing CD 20 membranous positivity in tumour cells (IHC 40x). Fig E\&F Showing Bcl 2 and CD 10 positivity in tumour cells (IHC 40x) Fig G, H, I showing CD3, Bcl6 and CD 5 negative, however native T cells exhibit positive staining. 


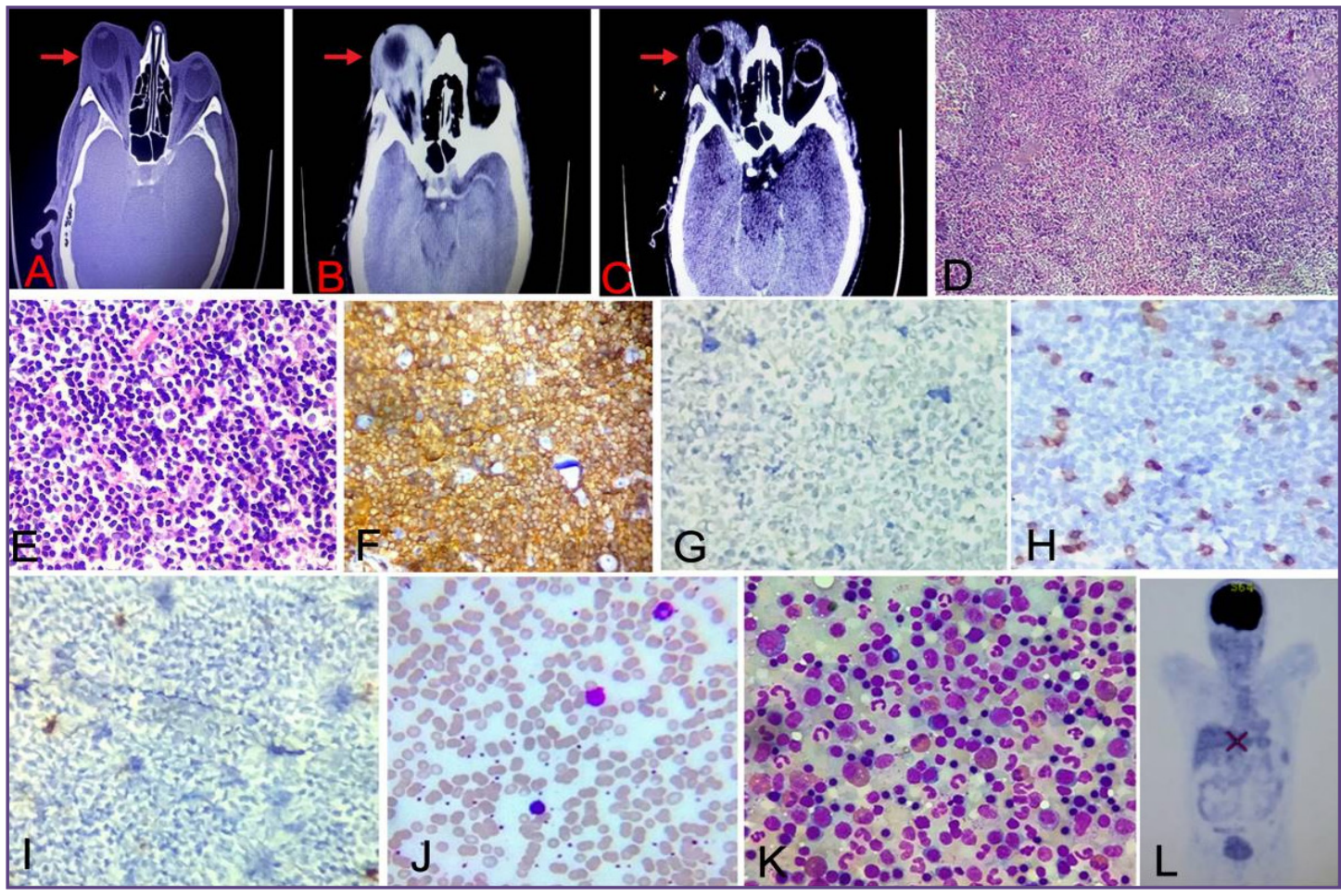

Fig. 2: (A, B \&C) CT Scan showing soft tissue mass in the right orbit. Fig D, E Microphotograph showing centrocytes and occasional Centroblasts in follicular pattern. (H\&E 10X,40X) Fig F-J: CD 20 positive, Negative for Bcl6, CD 10 and CD 03(IHC 40x). Fig J\& K: PS and BMA free of disease (Leishman 10x,40x.). Fig L: PET SCAN showing increased metabolic activity.

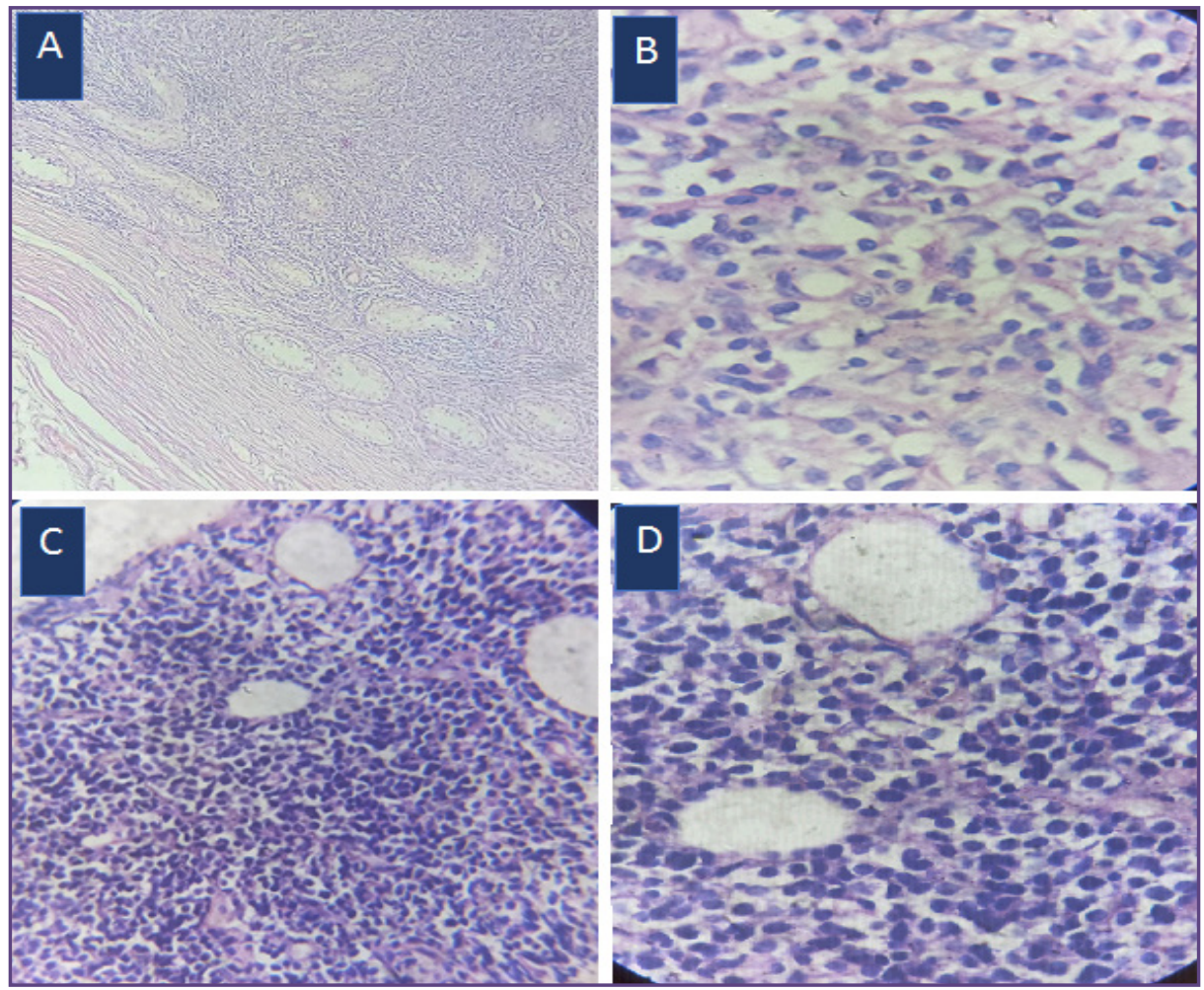

Fig. 3: A\&B showing microphotograph of testicular ENL, Fig C\&D microphotograph of Diffuse large cell lymphoma in breast. 


\section{FIG 4 :DISTRIBUTION OF ENL ACCORDING TO}

\section{THE SITE}

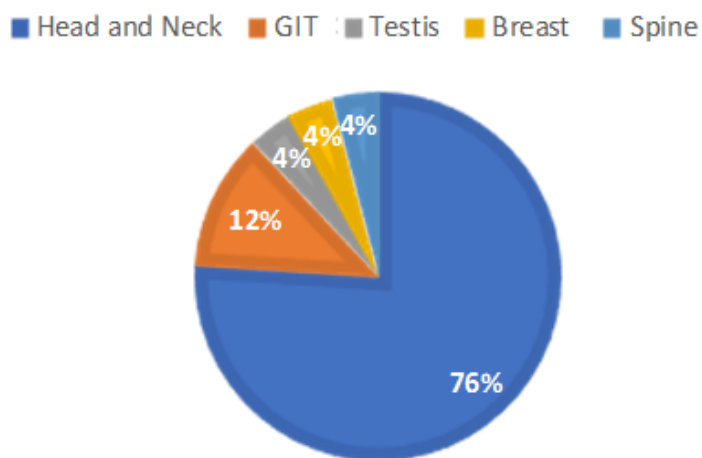

Fig. 4: Distribution of ENL according to the site.

tissue lymphoma (MALToma) or follicular lymphoma into DLBCL is known to occur. ${ }^{[1]}$ In the present study, all tonsillar lymphoma were of B cell subtype with diffuse large cell lymphoma in $80 \%$ cases (Fig 1). Microscopy shows tumor cells which are 4-5 times the size of a small lymphocyte in sheets having large round to oval vesicular nucleus, 2-3 prominent nucleoli and pale basophilic cytoplasm. Follicular B type seen in 2 cases (20\%). Histology of numerous hyperplastic follicles with germinal centre mimics reactive follicular hyperplasia. However, lack of polarization of germinal centers, negligible phagocytic activity and monomorphic cytology of neoplastic cleaved cells, prompts towards follicular lymphoma. IHC screening panel exhibits B cell phenotype in both follicular lymphoma and reactive lymphoid hyperplasia, but large number of reactive follicular cells switch off the expression of BCL-2 protein to undergo apoptosis ${ }^{[13]}$ in the latter. In the present case strong membranous/cytoplasmic staining for BCL-2 protein seen in our cases of follicular lymphoma.

Orbital lymphoma involves the ocular adnexa such as conjunctiva, eyelids, lacrimal glands or retroorbital soft tissue. Depending on anterior or posterior location of the orbital lesion, patients present with symptoms of blurred vision, diplopia, swelling, proptosis and rarely pain. Proptosis was the presenting complaint in all our cases. Fundoscopy and CT scan revealed retro-orbital mass. Usual histology is marginal zone B cell lymphoma of MALT type. ${ }^{[5]}$ DLBCL-NOS histology was seen in $50 \%$ of our study cases, while one case exhibited follicular B cell type of ENHL and the other one was Small lymphocytic lymphoma-B cell type (Fig 2).

Intraoral lymphomas constitute $25 \%$ of all lymphomas ${ }^{[14]}$ with gingiva being the commonest site. In our study we had one case in gingiva and 2 cases in oropharynx altogether constituting $15.8 \%$ cases. All the cases showed diffuse large cell lymphoma, and on IHC two were B cell and the other of T cell immunophenotype cell type of diffuse large cell lymphoma was seen in one case of paranasal sinus.

In the present study, 3 cases were seen in GIT and exhibited DLBCL-NOS. Two were seen in gastric region and one in the rectum. Both gastric lymphomas were negative for $H$. pylori on giemsa stain. H. pylori infection seen in $2 / 3$ cases of marginal zone lymphoma of MALT in GIT and tumor regresses following eradication of infection. [4,9] Considering the differential of carcinoma, additional IHC markers CK, EMA, chromogranin was done and were negative.

Testicular lymphomas comprise $5 \%$ of all testicular malignancy. It is the most common tumor in elderly and it has to be differentiated from seminoma and plasmacytoma. [15] In our study, 51-year male patient presented with swelling in the left testis. Preoperatively alpha-fetoprotein (AFP), lactate dehydrogenase (LDH) and beta-human chorionic gonadotrophin ( $\beta$-hCG) blood levels were normal. Cut surface of the orchidectomy specimen showed grey brown, firm to hard, tumor. Microscopy revealed diffuse sheets of polymorphic population of large cells, medium cells with vesicular nucleus having prominent nucleoli and small cells with scant cytoplasm. Large cells were multinucleated and the differential of spermatocytic seminoma was made on histopathology (Fig 3A,3B). On IHC, Pan CK was negative, CD45 and CD 20 was positive favoring a diagnosis of DLBCL-NOS.

The incidence of primary breast lymphoma ranges from $0.04-0.5 \%{ }^{[14]}$. Lymphomas usually lack the irregular borders of invasive carcinoma - NOS. Breast lymphomas in our study constituted $4 \%$ of cases. Microscopy of trucut 
biopsy showed monomorphic population of large, round to oval hyperchromatic cells in sheets (Fig 3C,3D). A diagnosis of Non-Hodgkin's lymphoma was made, and on IHC it was phenotypically subtyped as DLBCL-NOS.

Other histologic subtypes of mantle cell lymphoma, extra nodal marginal zone B-cell of MALT type are reported in literature. Sporadic cases of Burkitt's lymphoma, though rare, do occur more frequently than in nodal counterpart. ${ }^{[5]}$ These cases were not seen in our study, possibly due to geographic variation in molecular expression. ${ }^{[5]}$

\section{Conclusion}

Lymphomas in the extra nodal sites are rare but has the propensity to occur in any anatomic site. Hence a differential of lymphoma to be considered in extra nodal site. Histology with IHC enables to differentiate from poorly differentiated carcinoma as the treatment modalities and prognosis varies.

\section{Acknowledgements}

NIL

\section{Funding}

NIL

\section{Competing Interests}

NIL

\section{Reference}

1. Padhi S, Paul TR, Challa S, Prayaga AK, Rajappa S, Raghunadharao D, Sarangi R. Primary extra nodal non hodgkin lymphoma: a 5 year retrospective analysis. Asian pacific J cancer prev 2012; 13(10): 4889-4895.

2. Mishra P, Das S, Kar R, Jacob Se, Basu D. Primary extranodal non-Hodgkin lymphoma: a 3-year record-baseddescriptive study from a tertiary care center in southern India. Indian jpatholmicrobiol2015;58:296-300.

3. CortiM ,Villafane M, Bistmans A, Narbaitz M, Gilard L. Primary extranodal non-hodgkin lymphoma of the head and neck in patients with acquired immunodeficiency syndrome: a clinicopathologic study of 24 patients in a single hospital of infectious diseases in Argentina. Int. Arch Otorhinolaryngol $2014 ; 18: 3$

4. Guruwale S,Gore CR,Karia KM,Paranjape SP,Bardapurkar PR,Kumar H,Buch AC.Extranodal NON hodgkins lymphoma: A rare case series.Clin Cancer Investig J 2018;7:137-142.
5. VannataB, ZuccaE. Primary extranodalB-cell lymphoma: current concepts and treatment strategies. Chin clin oncol 2015:4(1):01-17.

6. Pai A, Kannan T, Balambika RG,Vasini V.A study of clinical profile of primary extranodal lymphomas in a teritiary care institute in south india. Indian J Med Paediatr Oncol 2017;38:251-255.

7. SimhaNV,Nitha, Sreenivas N. Primary Extranodal Non Hodgkins lymphoma of Left Tonsil: A case report. Journal of Evidence based medicine and Healthcare 2014; 1(12):14841488.

8. Payandeh M, Sadeghi M, Sadeghi E,Aeinfar M.The survival of extra nodal non-Hodgkin's lymphoma patients in the west of Iran.American journal of cancer prevention2015;3:96-99.

9. Goldlum J R, Lamps L W, McKenney JK, Myers JL.Rosai and Ackermans Surgical pathology.11th ed.Philadelphia:Elsevier;2018.

10. Mertsoylu H, Muallaoglu S, Besen A, Erdogdu S,SezerA, Sedef AM et al.Primary extranodal non-hodgkin's lymphoma: clinicopathological features, survival and treatment outcome in two cancer centers of southern turkey. Asian pac j cancer prev 2014; 15 (17):7207-7211.

11. Salplahta D, Comanescu M V,Anghelina F, Ioniţa E, Mogoanta C A, Anghelina L. Non-Hodgkin lymphomas of Waldeyer's ring. Rom j morpholembryol 2012, 53(4):10571060.

12. VasilakakiT, MyoteriD, TsavariA, SkafidaE, ArkoumaniE, KouliaK et al. Localised extranodal non-hodgkin's lymphoma of the tonsil: report of a rare case. OA case reports $2013 ; 2(11): 1-2$.

13. Harry L Ioachim, L Jeffery Medeiros. Iocham's Lymph node pathology. 4th edition. Philadelphia: Wolter Kluwer; 2009.391-400.

14. Aparna C, Renuka IV, RajaniM,Sailabala G, Annapurna P. Primary extranodal lymphomas-spectrum of distribution and morphology with immunophenotyping-Spectrum of distribution and morphology with immunophenotyping 3 year institutional study. Med J DY Univ2015;8:138-43.

15. Mohan H. Textbook of pathology. 6th ed. New Delhi: Jaypee brothers; 2010.C) Microphotograph of tonsil showing Diffuse large cell lymphoma (H\&E 10x,40x,100x). Fig D showing CD 20 membranous positivity in tumour cells (IHC 40x). Fig E\&F Showing Bcl 2 and CD 10 positivity in tumour cells (IHC 40x) Fig G, H, I showing $\mathrm{CD} 3, \mathrm{Bcl} 6$ and $\mathrm{CD} 5$ negative, however native $\mathrm{T}$ cells exhibit positive staining.

*Corresponding author:

Dr Netra M Sajjan, Assistant professor, Dept of Pathology, ESICMC \& PGIMSR, Bangalore

Email: sajjan.netra@gmail.com

Date of Submission $\quad: 04 / 09 / 2020$

Date of Final Revision : 10/01/2021

Date of Acceptance $\quad: 16 / 01 / 2021$

Financial or other Competing Interests: None.

Date of Publication : $28 / 02 / 2021$ 